\title{
LA PRESENZA DEI MERCANTI GENOVESI IN POLONIA NEI SECOLI XIV E XV
}

Introduzione

La presenza degli italiani in Polonia sin dal XIII secolo è abbastanza nota agli storici polacchi. A tale riguardo sono state effettuate diverse ricerche scientifiche che hanno trovato il loro apice a cavallo dei secoli XIX e XX, con studiosi come Jan Ptaśnik, Stanisław Kutrzeba e Marian Małowist che nei loro studi sui rapporti commerciali della Polonia con altri paesi nei secoli del tardo medioevo si sono imbattuti nella presenza di mercanti italiani di varia provenienza. Nelle loro ricerche questi studiosi hanno spesso trattato nel loro insieme i rapporti commerciali polacchi con i diversi stati in cui allora era frammentata la nostra penisola, oppure, nel caso delle ricerche di Danuta Quirini-Popławska, sono stati esposti i contatti commerciali della Polonia con le colonie genovesi del Mar Nero. La diffusa presenza della comunità genovese all'interno dello Stato polacco nei secoli XIV e XV offre caratteristiche proprie degne di essere raccolte e presentate in una dissertazione a parte.

\section{I primi mercanti}

La Polonia era conosciuta ai mercanti italiani fin dal Duecento. La genesi del loro afflusso presenta iniziali motivi di carattere fiscale-eclesiastico (Menotti Corvi 1927: 4). In quel tempo le province polacche erano solite pagare l'obolo di San Pietro che le poneva sotto la protezione della Santa Sede. Si trattava di una mossa di natura geo-politica, al fine di limitare la 
forte presenza dei commercianti tedeschi che stavano assumendo una posizione dominante all'interno del mercato locale. Un fattore di una certa rilevanza era la provenienza dei primi dignitari della chiesa polacca i quali erano, per la maggior parte, abitanti della penisola italiana (solitamente romani o toscani); risultava, dunque, naturale che la loro presenza suscitasse il richiamo di altre persone dalla stessa lingua e provenienza. I collettori della Camera Apostolica che si recavano a Cracovia affidavano le somme ai mercanti locali i quali, a loro volta, le consegnavano ai banchieri papali di Bruges e Venezia. Grazie al funzionamento di questo meccanismo tra la città fiamminga, Cracovia e alcuni centri urbani della penisola italiana iniziò a crearsi un solido rapporto commerciale, cui, sempre a fini commerciali, seguì una vera migrazione che ebbe il suo apice tra il 1333 e il 1434, anni durante i quali in Polonia si susseguirono i regni di Casimiro il Grande, Luigi i il Grande e Ladislao II Jagellone. Alla testa di questo fenomeno si posero i genovesi provenienti soprattutto dalla Superba, ma anche dalle colonie del Mar Nero (Ptaśnik 1910: 9).

In seguito, il flusso di italiani verso la Polonia subì un calo, per poi aumentare vertiginosamente nei primi anni del Cinquecento grazie alla regina italiana Bona Sforza, ma la presenza genovese non fu qui così significativa come in precedenza.

Bisogna, dunque, stabilire quali fossero le cause che suscitarono l'interesse dei liguri nei confronti di questo paese già nel XIV secolo. Si tratta, sostanzialmente, di due semplici motivazioni; la prima era di carattere logistico: l'allora favorevole posizione geografica faceva della Polonia, con a capo le città di Cracovia e Leopoli, un importante crocevia di traffici, soprattutto inteso come punto di passaggio terrestre per il congiungimento di centri di commercio che erano già nell'orbita genovese (Fiandre e Mar Nero). La seconda ragione era di interesse puramente commerciale e si sarebbe sviluppata solo in seguito; riguardava l'amministrazione, l'acquisizione e la vendita delle numerose materie prime di cui la Polonia disponeva in quel tempo.

\section{Un interessante punto di transito}

Il primo dei motivi dell'interesse genovese verso lo Stato polacco era, dunque, rafforzare la rotta per i traffici commerciali della Superba, già ben sviluppati sia a oriente che a occidente. In Europa occidentale la presenza di mercanti genovesi, insieme a quella di fiorentini e veneziani, era fortemen- 
te attestata nelle Fiandre. Gli accordi politico-commerciali con i genovesi risalgono alla metà del Trecento, quando essi vennero invitati a stabilirsi ad Anversa, ma alla fine scelsero come principale punto d'insediamento la città di Bruges (Petti Balbi 2003: 3-5). I privilegi di Anversa permisero comunque ai liguri la vendita al minuto, in città, di cibi e di tre qualità di birra, mentre a Bruges, allora il più importante centro commerciale di tutto il nord Europa, venivano importate materie tintoree come galla e allume (Belgrano, De Simoni: 529). In questa città i genovesi possedevano una numerosa colonia con un proprio fondaco e un consiglio direttivo, la masseria, a capo della quale vi erano un console e due consiglieri.

In Europa orientale i genovesi possedevano colonie sia nel Mediterraneo che nel Mar Nero; la più potente di esse era indubbiamente la città di Caffa, in Crimea, ma in questa penisola c'erano anche Vosporo, Soldaia, Alupka e Cherson, mentre sul mare d'Azov prosperavano Tana e Magreva. Dal punto di vista logistico le merci da commerciare partivano via mare da questi centri costieri, facendo tappa a Famagosta o in altri porti, arrivavano così a Genova, per poi eventualmente continuare verso le Fiandre. Con l'affermarsi della potenza turca nel Mar Nero le rotte marine diventavano sempre meno sicure, allora si decise di rinforzare la via commerciale terrestre, il cui migliore punto di passaggio erano i territori dello Stato polacco, soprattutto grazie alla conquista da parte di quest'ultimo della Rutenia Rossa (1340). I buoni rapporti commerciali e politici con la Polonia avrebbero, dunque, garantito il mantenimento di questa rotta commerciale che collegava Caffa e le altre colonie del Mar Nero con Bruges e il resto dell'Europa occidentale. Arrivata la merce a Cracovia, i mercanti genovesi, oltre a venderne parte in loco, potevano scegliere tra due percorsi, sempre verso occidente: il primo era una via orizzontale, cioè l'attraversamento degli stati di lingua tedesca, il secondo, in parte anche fluviale, passando da Toruń portava a Danzica, importante centro marinaro che faceva parte della Lega Anseatica e aveva stretti rapporti commerciali con le Fiandre. Le terre polacche del sud, con l'asse Leopoli - Cracovia - Breslavia, si trovavano quindi al crocevia di due importanti rotte commerciali che univano oriente e occidente d'Europa e, allo stesso tempo, il Baltico con il sud. Queste vie erano note ai mercanti cracoviani che già nel XIII secolo, tramite l'Ungheria, trasportavano le merci da vendere a Bruges. I genovesi adattarono questo percorso alle loro esigenze, facendo della via tra Caffa e Leopoli una rotta commerciale dove la loro presenza era dominante. La variante più battuta, via Kamieniec Podolski e Tarnopol, prese il nome di via tartarica. Da 
Cracovia, inoltre, partiva anche un percorso, chiamato via ordinaria, che, passando per Vienna, Bruck, Leoben Villach, Treviso, terminava a Venezia, collegando direttamente la penisola italiana alla Polonia. Grazie ai forti rapporti commerciali che Cracovia e Breslavia avevano anche con Norimberga, spesso si creava un'ulteriore variante attraverso la Baviera e il Brennero.

\section{L'ascesa di Leopoli e la presenza genovese}

Nel XIII secolo e agli inizi del XIV il centro polacco di commercio più importante per i coloni genovesi del Mar Nero era Włodzimierz Wołyński'. Durante il regno di Casimiro il Grande e il conseguente passaggio di questa regione alla Polonia, il ruolo di principale centro commerciale passò alla città Leopoli (Małowist 1947: 62). Stanisław Kutrzeba (Kutrzeba 1903: 156) scrive che Leopoli importava merci dall'oriente tramite le colonie genovesi della Crimea già dal XIII secolo, quando dei traffici commerciali si occupavano principalmente italiani, ebrei e tedeschi. Grazie ai buoni rapporti con Luigi i il Grande, a partire dal 1440 (Ptaśnik 1922: 51) la maggior parte dei genovesi si spostò dalla capitale Cracovia a Leopoli, allora in piena fioritura commerciale e geograficamente più vicina alle colonie del Mar Nero. La città divenne, proprio dopo Cracovia, il secondo importante centro polacco negli scambi commerciali. Oltre allimportante produzione della cera, Leopoli possedeva un importante mercato della seta, considerato secondo solo a quello di Venezia (Kutrzeba 2009: 292). Secondo alcuni storici polacchi, tra cui Stanisław Lewicki (Lewicki 1921: 20), la caduta di Costantinopoli (1453) e la chiusura del Bosforo ebbero un effetto benefico sul mercato di questa città, verso la quale si indirizzarono $i$ traffici in precedenza realizzati via mare. Dall'esempio genovese trassero vantaggio anche gli stessi abitanti della città; ad esempio Ptaśnik (Ptaśnik 1910a: 9) scrive di un armeno, un certo Tejčadin, residente a Leopoli, che possedeva magazzini per la merce non solo in città, ma anche nella stessa Caffa. In quegli anni, dunque, fino alla caduta di Caffa (Łożyński 1890: 71)

Leopoli era stazione genovese. L'epoca genovese di Leopoli fu la più florida, soprattutto dal punto di vista commerciale. Di essa si sono conservati alcuni frammenti di atti, ma in questi resti si possono ritrovare le tracce di come il commercio e la cultura genovese fossero fortemente legati alla società della

\footnotetext{
' Oggi Volodymyr-Volyns'kyj, in Ucraina.
} 
città di allora. I genovesi sono ospiti fissi a Leopoli, prendono parte a cause in tribunale, fanno accordi, stipulano contratti all'interno del municipio e in tutta la città dei leoni, siedono negli organi giudiziari, sebbene ci sia pervenuta solo una parte delle fonti di quel tempo,ci sono rimaste molte tracce di tale presenza (traduzione dell'autore dell'articolo).

I genovesi a Leopoli prendevano parte, sebbene in maniera minore, anche al commercio del cotone (Nam 2007: 379). Si trattava soprattutto di famiglie della Superba che risiedevano nella colonia di Pera, ma passavano alcuni periodi dell'anno proprio nella città polacca per vendere i loro prodotti. Nel XIV secolo la via tartarica (Quirini-Popławska 2002: 221) ${ }^{2}$, era praticamente l'unica che dal Mar Nero portava in Polonia, a Leopoli³. Oltre al commercio questa via servì anche alle ambascerie genovesi e veneziane proprio per recarsi in Crimea (Ibidem: 212).

Uno dei primi genovesi di cui si trova menzione negli archivi della città dei leoni è Francesco de Cantello, proveniente da Caffa, nominato cittadino di Leopoli nel 1409 (Sapori 1925: 138). Nel XV secolo molti genovesi iniziarono a stabilirsi nella Rutenia Rossa per curare affari in loco. Tra essi, oltre a de Cantello, si possono citare Messoporo de Ansaldo, Grimaldi, Angelo de Lercario, Cristoforo e Battisa Fragi, Barnaba de Negronio, Raffaele e Domenico de San Romolo, Giuliano e Antonio de Valetariis, Giuliano di Gibileto, Corrado de Portu, Lorenzo de Lomelli (Z Sali odczytowej 1921: 4). La maggior parte di loro si dedicò alla compravendita di merci, ma alcuni si occupavano anche dell'amministrazione delle dogane o delle miniere di sale dei dintorni di Drohobicz, Kołomyj, Przemyśl, Sanok e Dolina, arrivando gradualmente a sostituire gli ebrei ( $e$ in parte $i$ tedeschi) che fino ad allora avevano occupato posizioni di rilievo in questo settore. Nell'amministrazione delle miniere polacche si distinsero soprattutto le famiglie de Valetariis e Di San Remo (de San Romolo o Sancto Romulo). In questa famiglia il componente più in auge fu Cristoforo Guardia di San Remo, personalità molto attiva nell'economia della Rutenia Rossa. Dal 1443 Cristoforo svolse il ruolo di doganiere leopolese, nel 1454 divenne supparo delle mi-

${ }^{2}$ Le città più importanti interessate dalla via tartarica erano Perekop, Targowica, Koniecpol, Kamieniec Podolski, Tarnopol e Leopoli.

${ }^{3}$ Solo in seguito si sviluppò la via attraverso la Transilvania, ma, secondo Marian Małowist, oltre alla difficoltà di superare le montagne, i mercanti genovesi incontravano spesso predoni lungo il percorso. Ne è testimonianza l'uccisione di un mercante genovese riportata nel Litterarum dell'Archivio di Stato di Genova (Litterarum 1475, f. 261, n. 749) e citata dallo stesso Małowist (Małowist 1947: 63). 
niere di Przemyśl e di Drohobicz. Probabilmente Cristoforo si occupò anche della tratta degli schiavi. Infatti, Quirini-Popławska (Quirini-Popławska 2002: 215), rifacendosi agli atti civici di Leopoli, ci informa che nel gennaio del 1444 un certo Christoforus Italicus (con ogni probabilità si tratta proprio di Guardia de San Remo) comprò uno schiavo da Stanislao de Gora al prezzo di dieci grivne. Sembra, dunque, che il genovese acquistasse schiavi nella "Rus Czerwona", per poi trasportarli a Caffa, da dove avrebbero preso la via per il Mediterraneo.

Grazie agli archivi civici di Leopoli, dove è presente il suo testamento (Akta grodzkie 1867: 107), si viene a conoscenza del fatto che a Leopoli risiedeva anche "Barbanas de Negrorio, Italicus lanuensis4, fundator et dotator altaris Sancte Mariae Virginis Visitacionis". L'altare venne fondato dal mercante genovese il due gennaio 1464, giorno in cui egli divenne proprietario del villaggio di "Pekulouiczie" (Pikułowice), nella provincia di Leopoli. De Negrorio (o De Negronio) partecipava attivamente alla vita della città anche come consigliere.

Nelle fonti d'archivio raccolte dal 1453 in poi si trovano, in qualità di giudici leopolesi, i genovesi Cristoforo e Raffaello de Sancto Romolo, Durino Cathanius, Antonio de Valentharis e Giuliano de Gebeleto da Caffa.

Dal 1475 in poi i genovesi, in seguito alla caduta di Caffa, iniziarono lentamente a lasciare le terre della Rutenia Rossa. Il loro posto fu gradualmente preso da mercanti milanesi, veneti e toscani.

\section{II sale e gli appalti}

Come si è in parte accennato, un altro motivo di fondamentale importanza per la presenza dei genovesi in Polonia nel XIV secolo erano i giacimenti di sale. Dal punto di vista dell'organizzazione commerciale, per Venezia l'utilizzo di un intero bastimento per il trasporto del sale era un fatto assai raro. Genova, invece, possedeva una vera e propria flotta destinata a questo commercio. Per la Superba il sale restava un mezzo di scambio fondamentale nei suoi traffici che vedevano anche l'esportazione di pesce, allume, grano e altro. In questo contesto generale si inserisce l'interesse di Genova verso le terre meridionali polacche che, oltre a costituire una terra di transito sicura, iniziavano a essere appetibili anche in virtù della presenza di miniere di sale: "Trade was not their sole interest. For a long time they

\footnotetext{
"In una successiva edizione del 1878 viene invece riportato "laicus lanuensis". (Akty grodzkie: 1878: VII, 164).
} 
hel the leases of the royal salt mines in Bochnia and Wieliczka and invested in lead-mining in the region of Olkusz and Trzebinia" (Moyssey Postan 1987: 559). Una fonte di primaria importanza a cui rivolgersi erano dunque gli appalti per i redditi della Corona, tra cui in primis vi erano appunto le miniere di sale e le dogane. Negli antichi catasti cracoviani si trovano nomi di appaltatori italiani di saline, tra cui spiccano soprattutto veneziani e genovesi. II principe Leszek il Bianco concesse ai minatori stranieri un privilegio che permetteva loro di governare le miniere gestite in Polonia secondo le leggi e le usanze dei loro paesi di origine. In tale documento vengono citati i "romani" che agli inizi del XIII secolo si interessavano allo sfruttamento dei giacimenti minerari polacchi. Con questa espressione si era soliti indicare gli italiani provenienti soprattutto dalla Toscana e da Roma, per distinguerli dai tedeschi. Per i genovesi, invece, si utilizzava spesso il termine "gallicus" (Ptaśnik 1910b : 32). Tale fatto può creare incertezza sul numero preciso dei commercianti realmente provenienti dalla Superba, in quanto potrebbero essere confusi con altri di origine franca (Ptaśnik 1910a: 10). A complicare ulteriormente la situazione è il fatto che a volte essi ricevevano anche un soprannome tedesco. Per esempio il commerciante Eustauchio de Parentibus era chiamato von den Elten. La tesi di Ptaśnik, secondo cui l'appellativo "gallico" nel Duecento e nel Trecento riguardava anche gli italici del nord, soprattutto i genovesi, trova appoggio anche in Armando Sapori (Sapori 1925: 127-128).

In questo contesto storico-economico si inserisce la figura dello "żupnik", il suppario, importante carica allora ambita sia dai tedeschi che dagli italiani. II "supparius salis" era un funzionario nelle miniere di Wieliczka e di Bochnia, allora chiamate "żupy królewskie”, ovvero 'miniere reali'. Il termine deriva dal polacco medievale "żupa"s che significava 'miniera di sale,' 'salina'. Oggi, nella lingua polacca moderna, "żupa" ha assunto un significato storico, lasciando spazio al più generico "kopalnia" che ha appunto l'accezione generale di 'miniera', senza distinzione alcuna, a cui eventualmente si aggiunge il termine indicante il minerale estratto.

Nel XIII secolo il commercio di sale delle miniere di Wieliczka e Bochnia ebbe una grande fioritura; il sale estratto cominciò a prendere varie direzioni: a est verso la Rus, a sud in direzione della Moravia, a ovest verso la Slesia e a nord verso la Masuria dove era oggetto d'interesse degli stessi cavalieri

${ }^{5}$ Il dizionario monolingue polacco riporta: "w dawnej Polsce: kopalnia, głównie soli" ("Nell'antica Polonia: miniera, soprattutto di sale", traduzione dell'autore dell'articolo; Szymczak 1981, III, lemma żupa). 
teutonici che ne avevano compreso l'importanza economica. II commercio del sale era comunque limitato da editti restrittivi e si concentrava soprattutto nelle mani dei mercanti e degli amministratori di Wieliczka, Bochnia e Cracovia, i quali potevano liberamente comprare questo minerale nelle miniere, esportarlo e venderlo dove volevano. Per il commercio del sale Cracovia possedeva addirittura un mercato a sé stante (forum solis) e alcuni privilegi voluti dalla città stessa fecero sì che i commercianti dell'antica capitale polacca diventassero i dominatori incontrastati di questo commercio. Infatti, i libri contabili ci segnalano affari che registrano quote significative (Kutrzeba 1903: 326). II re Casimiro il Grande diede inizio a una riorganizzazione delle miniere, arrivando, nel 1368, a crearne una sorta di statuto con tradizioni e regole, dove vengono citati i passati suppari, il loro lavoro e i giuramenti. Gli storici polacchi sono concordi nel confermare che l'attività degli appaltatori italiani fu di fondamentale importanza per lo sviluppo dell'industria del sale. In particolare, Ptaśnik (Ptaśnik 1922: 25-38) riconosce ai genovesi il merito dell'organizzazione delle miniere, grazie a cui l'interesse per ottenerne i diritti allo sfruttamento aumentò a tal punto che si dovettero istituire gare d'appalto in cui si offrivano forti somme. I genovesi in quel periodo ottennero dunque il primato nell'industria mineraria, arrivando a superare $i$ tedeschi. In alcuni casi questi ultimi riuscirono ancora a ricevere qualche sporadico appalto nelle miniere dipendenti da Cracovia, ma quelle della Rutenia Rossa, ovvero Drohobicz, Dolina e Przemyśl, furono governate da suppari italiani che solitamente ricoprivano anche la carica di podestà del luogo, giocando un ruolo notevole nella legislazione mineraria del 1368. Tra i principali suppari, dunque, vengono rammentati proprio i genovesi. Alcuni appartenevano alla famiglia Cavallo, altri erano Goffredo Fattinanti “supparius generalis", Francesco di Cantello, aggregato alla nobiltà di Leopoli nel 1409, Giulio de Valentariis, deceduto ricchissimo nel 1468.

Il 23 marzo del 1454 il re diede in amministrazione le miniere di sale vicino a Przemyśl e Drohobicz a due genovesi, il citato Cristoforo de Sancto Romulo e Giuliano del Waleratiis; le prime per duemila grivne annuali, due pietre di pepe e una di zafferano secondo il peso di Leopoli, alla dogana della città sarebbero invece andate novecento grivne. Alla miniera di Drohobicz e alla sua dogana ne sarebbero spettate invece millequattrocento. Naturalmente c'era anche una tassa a titolo di concessione versata al re (Akty grodzkie 1867: IX, 76). Secondo Ptaśnik (Ptaśnik 1910b: 8) ciò è la conferma che allora i mercanti genovesi dovevano disporre di cifre davvero considerevoli, nella maggior parte dei casi investite con grande abilità. 
Un ulteriore commercio molto redditizio a cui Genova non voleva sottrarsi era la tratta degli schiavi, mercato spartito con la rivale Venezia. Spesso gli schiavi venivano mandati in Egitto, dove erano educati alla fede islamica e veniva insegnata loro l'arte della guerra, per poi diventare Mamelucchi. In altri casi essi venivano venduti nella penisola italiana e destinati a uso domestico. Siccome la maggior degli schiavi di cui si ha notizia nei registri era di provenienza orientale, si può facilmente intuire che i mercanti genovesi si procacciassero le loro vittime soprattutto nei dintorni delle colonie del Mar Nero (Fossati Raiteri 2008: 67), dove catturavano giovani autoctoni adatti alla vendita. Nella penisola italiana gli schiavi erano allora presenti in quasi tutte le città. In maggioranza si trattava di persone di origine africana, ma figuravano anche tartari, circassi, Ruteni, greci, nonché slavi orientali, chiamati spesso Sciiti o greci.

In Polonia il mercato degli schiavi era gestito in buona parte dagli italiani. Anche in questo caso, fino al XVI secolo i genovesi la fecero da padroni. Nel 1466 la città di Caffa ottenne dal re polacco uno speciale privilegio commerciale che permise ai suoi mercanti la vendita di schiavi in Polonia, ma anche, previo pagamento di dazi doganali, il passaggio sul territorio polacco di varie merci per poi portarle a Genova. In tale editto vengono anche citati gli schiavi; per ognuno di essi bisognava pagare un floren (Kutrzeba 1903: 321). Molti genovesi (come Agostino de Ferraris, Pietro Pallavicini, Pietro Lercario e il citato Cristoforo de San Remo) si occupavano del commercio degli schiavi, ma spesso delegavano altri mercanti genovesi sul territorio polacco.

\section{Alcuni mercanti genovesi a Cracovia}

Uno dei primi commercianti genovesi di cui si ha notizia in Polonia è Nicola Manente (Manentis, Mannete) Gallicus che fu anche borgomastro di Wieliczka. Nei documenti viene citato soprattutto come "Nicolao Gallicus dicto Manentis, cive Cracoviensi atque lanuensi" (Pekosiński 1887: 30) e il suo nome è legato alla miniera di "Veliczka". Manente arrivò in Polonia nel 1339, probabilmente insieme al fratello Marsonus. Dopo la fallita rivolta contro Ladislao i II Breve, Gerlach di Kulepn, borgomastro di Wieliczka, venne deposto dalla carica che nel 1306 fu appunto affidata a Manente il quale in seguito la vendette all'amico Mikołaj Wierzynek per la considere- 
vole cifra di millecento grivne. II mercante genovese godeva di una grande fiducia da parte del re Ladislao I, ricevendo il privilegio, nel 1324, di rappresentarlo in una missione di ambasceria ad Avignone a fianco del canonico cracoviano Piotr Miles. Sembra che la sua missione abbia avuto successo. Papa Giovanni XXII gli consegnò persino una bolla che giustificava la sua lunga assenza dalla Polonia. Si trattava con ogni probabilità di operazioni finanziarie, in quanto nel 1329 Nicola venne attestato, insieme al fratello, come mediatore bancario del collettore papale per la Polonia. Nonostante non si disponga di documenti precisi si può supporre che Manente fu supparo a Wieliczka, in quanto prima dell'incarico di Wierzynek gli archivi informano di un "Nicola supparo" negli anni 1313 e 1329, mercante e consigliere di Sandezia (Sącz). Proprio qui egli appare (sempre con il solo nome) in qualità di finalizzatore di una contesa commerciale tra Cracovia e Nowy Sącz.

Uno degli amministratori più famosi era il genovese Paulinus (o Paszko) Cavallo, chiamato anche Peterlin, supparo o sottosupparo di Bochnia, presente nei documenti cracoviani dal 1344 al 1358. Negli atti viene chiamato Gallicus. Probabilmente era arrivato in Polonia insieme al collettore della camera papale Galhard di Cahors, o con Andrea de Verolo come lo stesso Galhard scrive in una lettera di raccomandazione: "per Dominum Andream de Verulis et Paulinum Cavallo de Genua" (Theiner 1861: 416). Ancora nel 1346 Paulinus sottolinea i legami con la sua città natale, firmandosi "civis Januensis et Cracoviensies" (Ptaśnik 1910a: 12). Nella storia di Bochnia viene ricordato per aver dato inizio alla costruzione del primo ospedale e per aver trasmesso su carta usi e costumi dei suppari. Nell'amministrazione della miniera si distinse anche Giovanni Cavallo, figlio di Paulino.

A Cracovia abitava il commerciante e supparo genovese Gotfryd (Goffredo) Gallicus, spesso registrato come Fattinanti o, nella forma latinizzante, Factinanti (Mühle 2014: 347), personalità di grande importanza nella capitale polacca, in quanto fu nominato anche consigliere cittadino. Ptaśnik (Ptaśnik 1922: 32) riporta che nel 1368 Goffredo, in qualità di amministratore delle miniere di sale, aiutò il re nella stesura di un loro ordinamento giuridico. Dei suoi interessi in città si ha traccia già dal 1366, ma solo un anno più tardi viene registrato come suppario di Bochnia, successore di Pietro Cavallo, con la dimora in una casa di via Szpitalna a Cracovia. Nei documenti cittadini il suo nome appare spesso, ma solo una seconda volta, nel 1369, con la carica di suppario (Ptaśnik 1922: 32). Nel suo testamento si trova l'informazione che Goffredo aveva avuto l'occasione di ammini- 
strare le miniere di sale durante tre regni: di Casimiro il Grande, Luigi i il Grande e Ladislao Jagellone. Grazie all'atto di vendita del 1393 di uno dei suoi palazzi all'interno delle mura di Cracovia si evince che veniva chiamato "Zupparius salis generalis Bochne et Wieliczcze" (Ptaśnik 1922: 33), nomina riconfermata nello stesso testamento in cui Dominus Sulko viene citato come "executorum, tutorum et fideicommisariorum testamenti et ultime voluntatis condam famosi viri domini Gothifridi Fattinanti de Janua, supparii salis generalis Bochne et Wieliczcze"6. II 15 dicembre 1392 Fattinanti designò Piotr Wysz e Spytek di Melsztyn esecutori materiali del suo testamento (Ozóg 1997: I, 16). Il documento in questione venne redatto sempre a Cracovia e in esso veniamo informati che il re gli è debitore di milleduecentocinque marc gross i quali dovranno essere dati alla "Premiliensis cathedralis ecclesia contra schimaticos" (Ptaśnik 1910c: 7). Fattinanti dimostra la sua profonda fede cattolica, ma non è scevro dal lasciare una parte della sua fortuna ai minatori.

Spesso il mercante genovese aveva bisogno di rappresentanti in Polonia, oppure nella stessa Genova se si trovava all'estero. Si trattava a volte di persone fidate, spesso di famigliari. Gli interessi di Domenico e Cristoforo de San Romolo erano curati dal loro padre Pellegrino che nella Superba rivendeva le pellicce di zibellino comprate dai figli in Polonia. Lo stesso accadeva per la famiglia Promontorio: i fratelli Paolo e Stefano amministravano i loro affari a Poznań, mentre il loro anziano padre mandava a Paolo la merce da vendere.

\section{I genovesi a Poznań}

Anche a Poznań, capoluogo della Wielkopolska, la presenza italiana fu significativa. Ne scrissero a riguardo, negli anni Venti, Wicherkiewiczowa, Groth e soprattutto Kaczmarczyk. Anche nel caso di questa città, nel $\mathrm{XV}$ secolo la comunità italiana più numerosa era quella dei genovesi. Tra essi si annoverano Paolo e Stefano de Promontorio, Peregrinus de Promontorio, Agostino Mazoni de Promontorio, Eustachio de Parentibus, Nicola di Noli, Gian Antonio de Insula e Antonio de Pino.

I primi mercanti italiani di cui si ha traccia sono proprio due genovesi, i fratelli Paolo e Stefano de Promontorio chiamati "italici" (Wicherkiewiczowa 1929: 173). Si tratta dei primi mercanti italiani di una certa impor-

\footnotetext{
${ }^{6}$ Archiwum Aktów Dawnych Miasta Krakowa. Ludicium feria sexta proxima ante festum sancte Margarethe (11 Julii 1393).
} 
tanza che gli atti cittadini di Poznań rammentino (Wicherkiewiczowa 1929: 173). Essi commerciavano in vari tessuti, tra cui primeggiava il damasco. Paolo de Promontorio, registrato anche come Italicus, de Janua, Walen, de Prementorio, era annoverato tra i più ricchi mercanti dello stato polacco-lituano. Era arrivato in Wielkopolska nel 1483 su raccomandazione del generale della Grande Polonia, Mikołaj di Kutno. Nel 1495 Paolo entrò a far parte del consiglio cittadino dove acquistò il diritto di "Civis posnaniensis" (Wicherkiewiczowa 1926: 4). I due fratelli riuscirono ad acquistare una palazzina al numero cinquantuno della Piazza del Mercato. Successivamente iniziarono i problemi. La merce ordinata da acquirenti tedeschi tardava ad arrivare, una parte della rotta terrestre era ormai territorio turco e spesso i trasporti venivano depredati. Fu così che la famiglia Promontorio accumulò debiti per poi cadere in rovina. Essendo morti senza eredi, l'immobile divenne direttamente proprietà del re che poi lo cedette a un altro italiano, il più famoso Filippo Bonaccorsi, in arte Callimaco: “Domus murata Philippi Callimachi quam regia majestat post Paulum Italicum illi donat (Wicherkiewiczowa 1926: 4).

Un altro mercante genovese che abitava a Poznań era un certo Agostino Mazoni, arrivato in città nel 1503 . Anch'egli ebbe problemi e processi, ma ne uscì quasi indenne, arrivando a sposare la figlia del ricco mercante Jan Krypa.

Si ha anche notizia di Peregrinus de Promontorio, nei documenti chiamato anche Peregrinus lanuensis, Italus de Permontorio, Italicus. Costui, dal 1494, era al servizio presso Paolo per il quale spesso curava i suoi affari a Norimberga (Acta consularia Posnaniensia 1494-1507: K2) e di cui segui in parte i destini. Infatti, dopo la bancarotta di quest'ultimo, anch'egli sembra scomparire dai documenti cittadini, per poi riapparire soltanto nel 1517 (Kaczmarczyk 1990: 13). Dagli atti processuali in cui questo mercante era coinvolto si evincono i suoi intensi rapporti commerciali con l'allora capitale Cracovia (Kaczmarczyk 1990: 14).

Nel 1503 viene registrata la presenza di Agostino (Augustyn) Mazoni de Promontorio, chiamato anche Italicus, Italus, Włoch, der Wahle, Prementoriensis de lanua, lanuensis de Posnania, Masonus. Anche nel suo caso sono presenti atti processuali riguardanti la vendita di merce, ma anche i suoi precedenti contatti commerciali con le città di Vilna e Cracovia quando, prima di insediarsi a Poznań, aveva una società con Eustachio de Parentibus. A Poznań egli si occupava della vendita di pelli di animali, viaggiando comunque in tutta Europa. Nonostante i debiti accumulati e alcuni 
sotterfugi al limite del legale, Mazoni viene annoverato tra i più importanti mercanti posnaniani del XVI secolo (Kaczmarczyk 1990: 17), riuscendo a intavolare commerci sia con l'occidente (soprattutto Norimberga) sia con l'oriente (Brèst), così come a nord (Danzica). Nella Polonia orientale ebbe ottimi contatti (in seguito diventati conflitti) con i commercianti della comunità ebraica. A Poznań, Agostino riusci ad acquistare alcuni palazzi, tra cui uno sulla Piazza del Mercato. Anche il suo matrimonio fu la conferma della sua posizione tra i ricchi mercanti della città: sua moglie Anna era figlia del ricco mercante Jan Krypa e di Jadwiga Blasing. Anna in seguito prenderà l'appellativo di "Włochowa".

Interessante è il caso di Niccolò de Noali, figlio di Paolo, proveniente da un villaggio vicino a Genova, nei documenti chiamato Ripparoli (Kaczmarczyk 1990: 26), l'odierna Rivarolo. Niccolò, oltre ad aver fatto arrivare Andreoli Guasco de Soldaia, amministratore della provincia di Gnieźno, firmò un contratto come giardiniere di Urjel Górka, vescovo di Poznań, dove, oltre al pagamento che avrebbe ricevuto (otto ducati e una lira genovese, più vitto e alloggio), si leggono le sue mansioni, ovvero la cura dei latifondi del vescovo e, soprattutto, "plantandi vineas causa" (Ptaśnik 1910a: 51).

Nel 1503 fece la sua comparsa a Poznań anche Antonio de Pino, chiamato anche Antonius Italicus, De Pynus, Walhe von Janua. Kaczmarczyk (Kaczmarczyk 1990: 27) ci informa che negli atti civici il suo nome appare soprattutto riguardo ai debiti contratti e che spesso non aveva intenzione di pagare. II mercante genovese si occupava soprattutto della vendita di seta e di pellicce. Successivamente arrivò a Poznań anche il citato Eustachio de Parentibus che nel 1502 aveva ricevuto i diritti di cittadino di Cracovia. I suoi contatti commerciali si sviluppavano da Poznań a Vilna, Glogau e Breslavia. Spesso le sue società erano con italiani, per esempio Augustinus Mazoni, ma lo troviamo anche con Fryderyk Boner, monetarius di Glogau.

Poco si sa invece di Baptista Walh Dologes von Janua, mercante che dimorava a Poznań, ma forse non in pianta stabile. Dai documenti si ha notizia di un suo scambio di merci con il mercante Karol Holzschuher. Sono annotati la cannella, lo zafferano e altre spezie. Le grandi quote di denaro di cui disponeva, segnalate nei registri civici, indicano che si trattava di un mercante attendibile.

ᄀ "Moglie dell'italiano" (trad. dell'autore dell'articolo). 
Per quanto riguarda gli appellativi ricevuti dai genovesi (e probabilmente anche dagli altri italiani) nei documenti riguardanti i genovesi residenti a Poznań si riscontra la netta prevalenza dell'utilizzo del termine "walh" o "walhe" rispetto a "italicus". II termine, proveniente dallo slavo ecclesiastico-antico, allinizio indicava "i popoli romani insediati a sud delle popolazioni slave" (Widtak, 2010: 19).

Tra gli italiani che nel XVI secolo a Poznań ricevettero i diritti di cittadinanza figurano soltanto i seguenti genovesi: Eustachius de Parentibus, dignitario di Sigismondo a Glogau, Jan Bernard de Sancto Petro e Angelo Maria Gandusso, mentre iniziava a essere alto il numero di fiorentini, lucchesi e veneziani. L'assetto geopolitico era cambiato e ciò si lo si percepiva anche a Poznań.

\section{La fine di Caffa e le ambascerie genovesi in Polonia}

I contatti commerciali tra la colonia genovese di Caffa e la Polonia erano dunque intensi, $\mathrm{ma}$, in seguito alla situazione resa instabile dalle pressioni turche e tartare, essi divennero anche di tipo diplomatico. Nel 1462 i vertici di Caffa arrivarono a chiedere al sovrano polacco Casimiro IV Jagellone lo Status di protettorato polacco. Prima di recarsi a Roma una delegazione di Caffa soggiornò a Cracovia (Quirini-Popławska 1995: 554) ${ }^{8}$. Negli anni successivi i rapporti della Polonia con Caffa furono intensi: non solo i genovesi si recavano in Polonia per commerciare, ma anche alcuni polacchi vivevano e lavoravano nella colonia genovese del mar Nero.

Nel gennaio del 1471 in Polonia arrivarono Giuliano Fieschi da Caffa e Giovanni Giambone da Genova, portando con sé due bolle del papa Giulio II in cui il pontefice concedeva la sua indulgenza a chi avesse finanziato la difesa di Caffa (Ibidem: 558). Le colonie sul Mar Nero erano sempre più minacciate dall'espansionismo ottomano, si cercavano quindi possibili alleanze per un eventuale protettorato. A tal scopo, nel marzo del 1474 arrivò a Cracovia l'ambasciatore della Repubblica di Genova Giuliano Gentile, mentre il doge di Genova, Paolo Campofregoso, inviò una lettera di raccomandazione a Casimiro Jagellone. Un anno più tardi, sempre a marzo, venne mandata un'altra ambasceria con a capo nuovamente Giuliano Gentile, in qualità di console di Caffa. A riguardo, nell'archivio di Stato di Genova si

${ }^{8}$ Danuta Quirini-Poplawska ne trova traccia in una nota del manoscritto di Sebastian Petrycy di Plzeň (Sebastianus Petricius Pilsnianus), professore presso l'Accademia di Cracovia. 
sono conservate le lettere di raccomandazione del doge di Genova inviate al "rex Poloniae" (Archivio Segreto Litterarum 23/1799, 1461-1484: 950) (Archivio Segreto Litterarium 24/A/1800/a, 1474-1478: v 10), all'imperatore tedesco e alle autorità di Cracovia.

La caduta di Caffa si ripercosse sui traffici commerciali polacchi con l'oriente i quali, come conseguenza, subirono un forte indebolimento. Alla dinastia polacca, però, non interessava un conflitto con la potenza turca; la politica della Polonia di allora era, purtroppo per Genova, più orientata verso i territori della corona della Boemia.

Ancora tra il 1483 e il 1484 il genovese Vincenzo de Domenico si trovava in Polonia per proporre la creazione di una lega antiturca (Ptaśnik 1922: 85). Caffa, però, era già caduta e, di conseguenza, per i genovesi la Polonia stava progressivamente perdendo la sua importanza strategica.

\section{Conclusioni}

Le informazioni d'archivio e quelle riportate dagli storici polacchi riguardanti la presenza e le attività genovesi in Polonia nei secoli XIV e XV sono numerose e per tale motivo necessitavano di uno studio ad hoc per fornire una visione d'insieme. Sono stati qui esposti i motivi per i quali i genovesi si recarono in Polonia nei secoli succitati, è stato sottolineato il loro dominio commerciale così come sono state introdotte alcune personalità di spicco in tre diverse città. Nei documenti analizzati e qui riuniti si ha la conferma dell'importanza di Leopoli, tappa della via tartarica da e per Caffa, della vendita delle merci trattate dai genovesi, ma anche della loro presenza sia nelle miniere di sale che come parte integrante della società polacca di allora, nella quali ricoprivano cariche pubbliche. La caduta di Caffa si rivela cruciale per il tramonto della presenza dei genovesi in Polonia che in parte rimarranno a Cracovia e poi a Poznań per poi lentamente lasciare spazio a mercanti di altre città della penisola italiana come Lucca, Firenze e Venezia. Risulta rilevante il fatto che, a differenza di toscani e veneti, i genovesi non proposero personalità nell'ambito artistico o in quello dell'insegnamento, campi in cui in seguito molti italiani arrivati in Polonia si sarebbero invece distinti. I cittadini della Superba, invece, confermarono il loro storico ruolo di pionieri e mercanti. 


\section{Bibliografia}

1867. Akta grodzkie i ziemskie z czasów Rzeczypospolitej Polskiej, z Archiwum tak zwanego Bernardińskiego we Lwowie, Galicyjski widział Krajowy, vol. VI, Leopoli: LXXV.

1878. Akta grodzkie i ziemskie z czasów Rzeczypospolitej Polskiej, z Archiwum tak zwanego Bernardińskiego we Lwowie, Galicyjski widział Krajowy, vol. VII, Leopoli: $X C$.

De Simoni C., Belgrano L. T. (a C. di), Documenti ed estratti inediti o poco noti riguardanti la storia del commercio e della marina ligure (data mancante).

Fossati Raiteri S. 2008. i genovesi e il mercato degli schiavi nel Vicino Oriente (sec. $X I V-X V I)$, "Rime" 1: 67-75.

Lewicki S. 1991. Targi Iwowskie od XIV do X IX wieku, Leopoli.

Łożyński W. 1890. Patrycyat i mieszczaństwo Iwowskie w XVI i XVII wieku, Leopoli.

Kaczmarczyk K. 1990. Włosi w Poznaniu na przełomie XV i XVI wieku, “Kronika miasta Poznania" 3/4: 131-159.

Małowist M. 1947. Kaffa - kolonia genueńska na Krymie i problem wschodni w latach 1435-1475, Varsavia.

Menotti Corvi A. 1927. Tradizioni storiche dei rapporti economici italo-polacchi, "PoIonia - Italia" 1: 3-13.

Moyssey Postan M. (a c. di) 1987. The Cambridge Economic History of Europe: The agrarian life of the Middle Ages, vol. II, Cambridge.

Mühle E. 2014. Breslau und Krakau im hohen und späten Mittelalter: Stadtgestalt, Köln, Weimar, Wien.

Jong-Kuk N. 2007. Le commerce du coton en Meditérranée à la fin du Moyen Age, Leiden - Boston.

Ozóg K. 1997. Spór o biskupstwo krakowskie w roku 1392 na tle sojuszników Polski z Papiestwem u schyłku XIV, "Kwartalnik Historyczny" CIV, vol. I: 3-20.

Petti Balbi G. 2003. i rapporti tra Genova e il mondo fiammingo, [in:] Primitivi: fiamminghi in Liguria, a c. di Cavalli Traverso C., http://www.rmoa.unina.it/1387/1/ RM-Petti\%20Balbi-Rapporti.pdf (consultato il 10.06.2017).

Piekosiński F. 1887. Kodeks Małopolski, vol. III, Cracovia.

Pistarino G. 1993. Pagine sul medioevo a Genova e in Liguria, Genova.

Ptaśnik J. 1910a. Włoski Kraków za Kazimierza Wielkiego i Władysława Jagiełty, Cracovia.

Ptaśnik J. 1910b. Z dziejów krakowskiego kupiectwa od XIV do XIX wieku, Cracovia.

Ptaśnik J, 1910c. Italia Mercatoria, Roma.

Ptaśnik J. 1922. Kultura włoska wieków średnich w Polsce, Varsavia.

Quirini-Popławska D. 2002. Włoski handel czarnomorskimi niewolnikami w późnym średniowieczu, Cracovia. 
Quirini-Popławska D. 1995. Z powiq̨zań Polski z Kaffa, koloniq genueńskq na Krymie w drugiej połowie XV wieku, [in:] Cracovia, Polonia, Europa, a c. di Baczkowski K., Bukowski W., Cracovia: 548-560.

Sapori A. 1925. Gl'italiani in Polonia nel Medioevo, "Archivio storico italiano" serie 7, vol. 3: 125-155.

Szymczak M. (a c. di). 1981. Słownik języka polskiego, vol. III, Varsavia.

Theiner A. 1861. Vetera Monumenta Poloniae et Lithuaniae, vol. I.

1921, 22 febbraio. Z Sali odczytowej, "Gazeta Lwowska" 42: 4.

Wicherkiewiczowa M. 1926, 13 aprile. Włosi w Poznaniu, "Dziennik Poznański” 84: 4.

Wicherkiewiczowa M. 1929. Wpływy kultury italskiej na Poznań, "Polonia - Italia” 5-6: 172-176.

Widłak S. 2010, Italia e Polonia, popoli e lingue in contatto, Cracovia.

\section{Archivistica}

Acta consularia Posnaniensia, K2 1494-1507.

(Genova) Archivio Segreto Litterarium, 23/1799: 1461-1484.

(Genova) Archivio Segreto Litterarium, 24/A/1800/a: 1474-1478.

\section{Abstract}

The presence of Genoese merchants in Poland in the 14th and 15th century

This article focuses on the exposition of the presence of the Genoese community in Poland in the 14th and 15th century. The Italian presence in Poland has been the object of historical research by various scholars, especially at the turn of the 19th and 20th century, but the various communities of the Italian peninsula have almost always been treated as a whole. The article focuses instead on the Genoese community and aims to bring together more information about it: the reasons that have pushed the citizens of La Superba to settle as first in Poland, the geopolitical situation, the types of trade carried out, the Polish urban centers involved in this immigration and the most popular personalities.

Keywords: Republic of Genoa, Genoese merchants in Poland, Genoese people in 14th and 15th century, Genoese people in Leopolis and Krakow, Italians in Poland Parole chiave: Repubblica di Genova, mercanti genovesi in Polonia, genovesi nei secoli XIV e XV, genovesi a Leopoli e Cracovia, italiani in Polonia. 